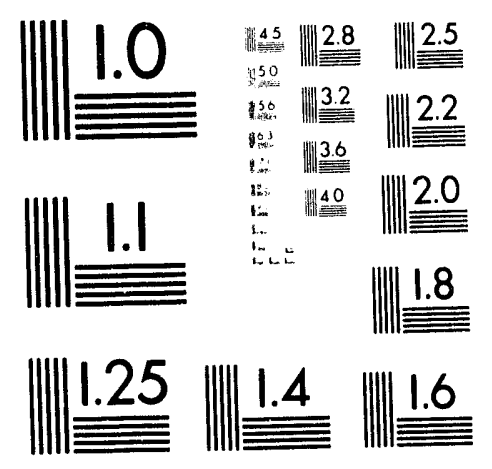



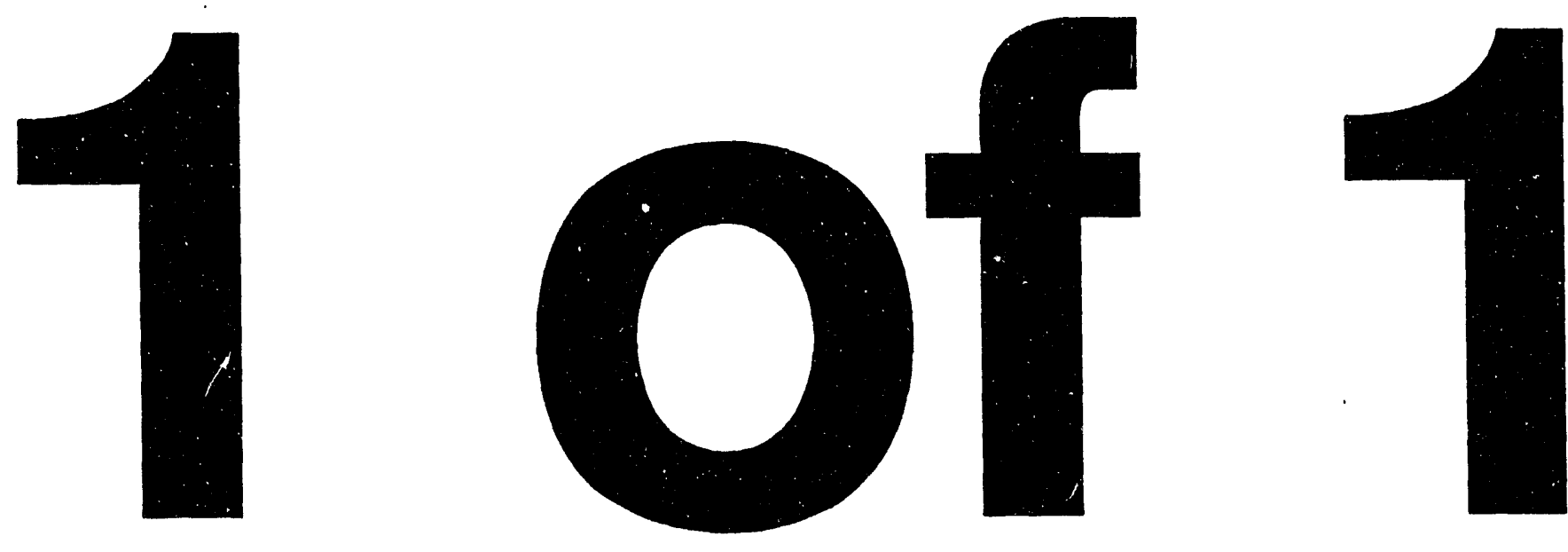
Submitted to Workshop on the

Stability of Particle Motion in Storage Rings

Brookhaven National Laboratory

October 19-24, 1992

\section{A SIMULATION (OF MODULATIONAL DIFFUSION FOR THE FERMILAB TEVATRON}

T. Satogata

Northwestern University, Evanston, IL 60205

and

Fermi National Accelerator Laboratory, Batavia, IL 60510

S. Peggs*

Brookhaven National Laboratory, Upton, NY 11973

\section{ABSTRACT}

A summary of the requirements for modulational (thick-layer) diffusion to exist in a particle synchrotron is presented and applied to a simple tune-modulated collider model of the Fermilab Tevatron where the only nonlinearities present are iwo beam-beam kicks. For certain realistic tune modulation parameters and single-particle base tunes, amplitude growth is observed over timescales appropriate to diffusive models. The character of this growth has qualitative features that are similar to those predicted by modulational diffusion models, but is significantly different in that the amplitude growth is exponential in time, not root-time as in classical diffusion. Some possible explanations for this effect are briefly noted, and impact of the possible existence of such a mechanism on future Fermilab collider upgrades is mentioned.

\section{CHARACTERISTICS OF MODULATIONAL DIFFUSION}

Modulational diffusion has been the subject of many investigations in the past ten years, since it provides a particle loss mechanism in many-dimensional dynamical systems such as particle accelerators over timescales that are longer than those from pure resonant loss (typically hundreds of turns), but shorter than the timescales of Arnol'd, or thin-layer, diffusion (typically hundreds of millions of turns). Most of the salient features and quantitative analysis can be found in assorted publications ${ }^{1-3}$; here we highlight the requirements for modulational diffusion to exist in a synchrotron and what characteristics such diffusion might have.

First, consider motion of resonant particles in only the horizontal transverse dimension. It has been shown that with a suitable variation of particle tune with amplitude, and within a one-dimensional resonance influenced by tune modulation, particle trajectories can be characterized as either regular or stochastic. ${ }^{4-6}$

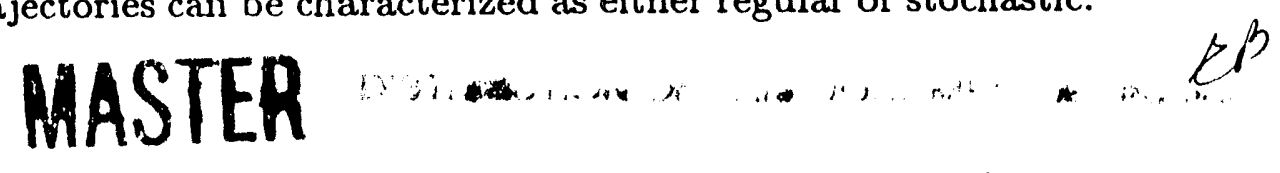

*Work performed under the auspices of the U.S. Department of Energy. 


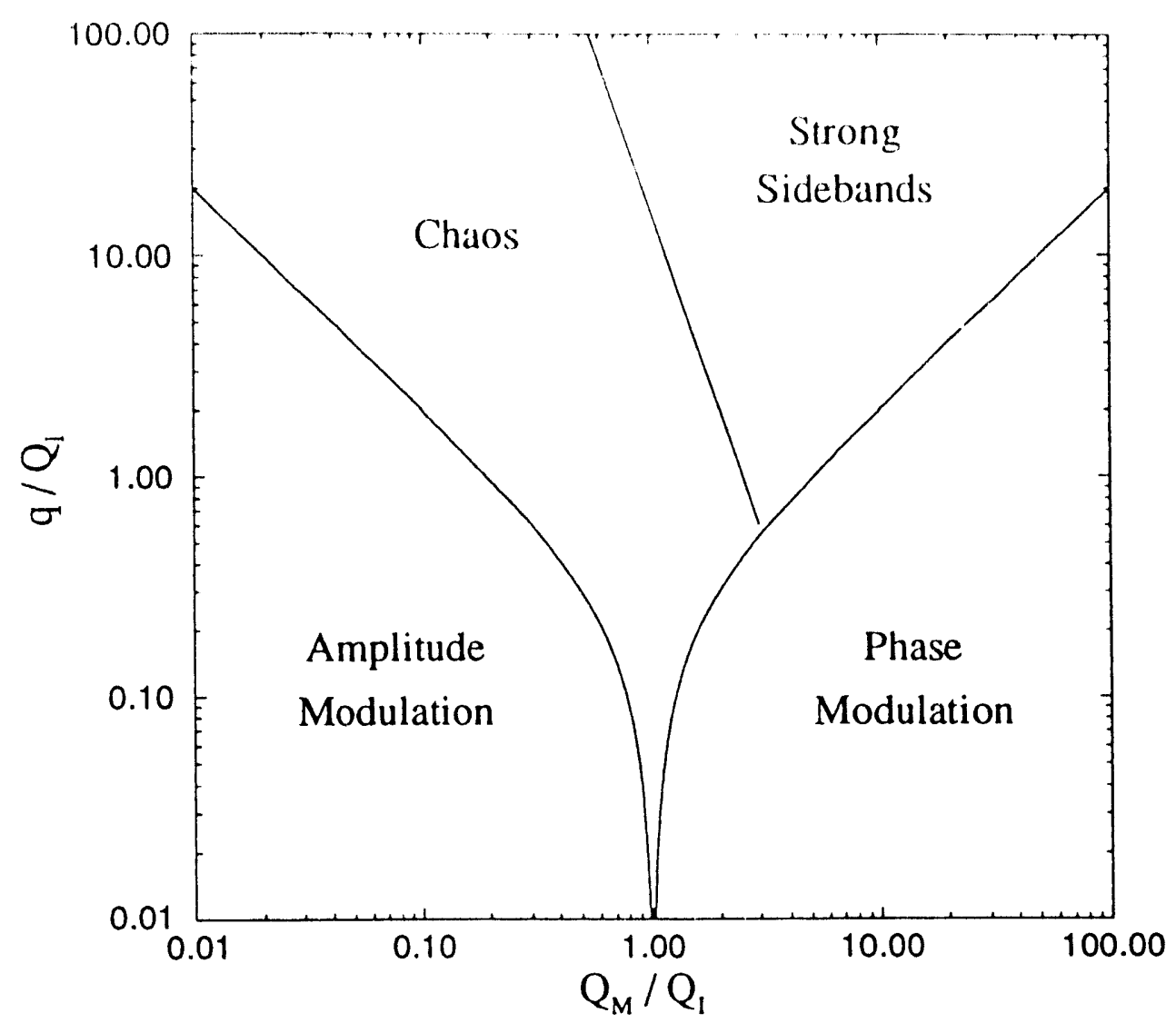

Figure 1: The tune modulation $\left(q, Q_{M}\right)$ parameter plane.

This is most clearly seen within a single-resonance tume-modulation model that produces four phases within the dynamical tune modulation parameter space $\left(q, Q_{M}\right)$, where the tune modulation is given by

$$
Q_{x}=Q_{x 0}+q \sin \left(2 \pi Q_{M} t\right)
$$

$t$ is time measured in machine turns, and $Q_{x 0}$ is the unperturbed horizontal base tune. (See Figure 1.) Note that in this figure both $q$ and $Q_{M}$ scale with the parameter $Q_{I}$, which is the frequency of small oscillations around locally stable fixed points inside the unmodulated resonance islands, or the so-called "island tune".

The relevant portion of this parameter space for modulational diffusion is the region marked "Chaos" - in this region sidebands around the primary resonance which are created by the tune modulation overlap (the so-called Chirikov overlap), and a thick layer of bounded chaotic motion is formed for appropriate tune modulation strength $q$ and frequency $Q_{M}$. (See Figure 2 - this is a stark 

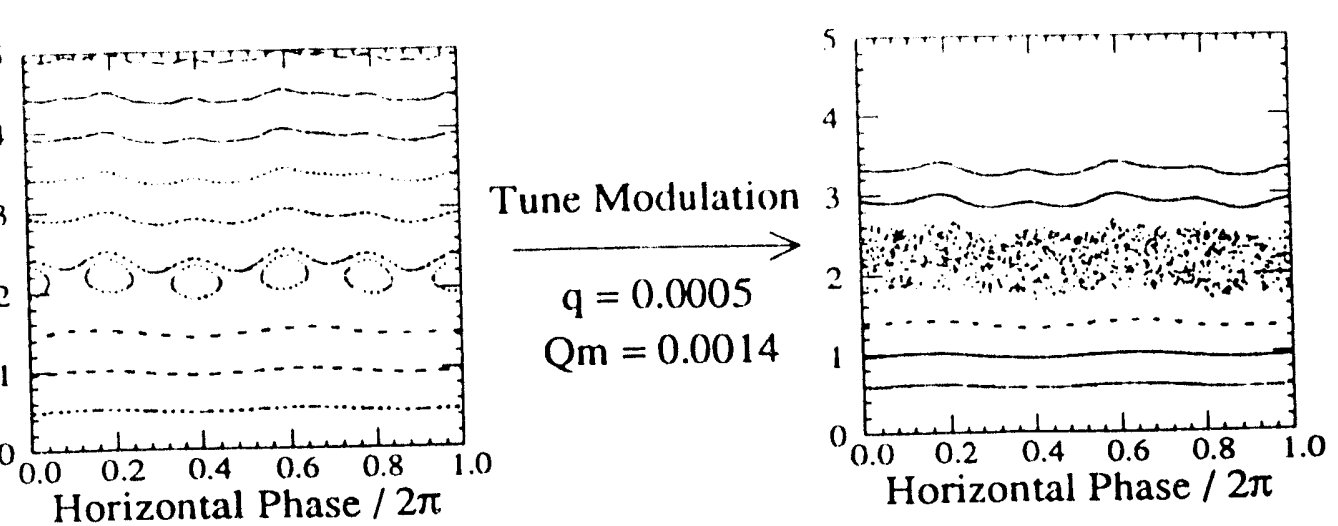

Figure 2: 1-dimensional phase space on the $Q_{x}=3 / 5$ resonance, for moderately realistic parameters in the Fermilab Tevatron. Particle tracking is done with the beam-beam B0D0 lattice, with a linear beam-beam tuneshift of 0.0005 . Resonance island structures are apperent without tune modulation, and tune modulation creates a thick stochastic band.

contrast to the thin-layer stochasticity generated by webs of interacting higherorder resonances seen in Arnol'd diffusion. ${ }^{3}$ ) In modulational diffusion models this layer of chaos serves as a noise source for vertical motion that is coupled to the horizontal phase through a weak nonlinear coupling resonance.

Examine what would be nominally regular motion in the vertical dimension, influenced by one or more of these "weak" coupling resonances. (For these purposes such resonances are considered to be "weak" if their amplitudes are much smaller than that of the primary resonance in the horizontal plane that drives the horizontal stochasticity.) The motion in this plane is now that of a very weakly driven oscillator, where the driving force is chaotic due to the weak coupling to the horizontal stochastic motion. Such motion is similar to that of a random-walk problem; the stochastically driven vertical motion can "diffuse" out to large amplitudes in finite time and therefore be lost from the machine.

The Hamiltonian for the standard modulational diffusion model ${ }^{1}$ can be written as

$$
\begin{aligned}
H_{1}\left(\theta_{x}, I_{x}, \theta_{y}, I_{y}\right)= & \frac{1}{2} I_{x}^{2}-\epsilon \cos \left[(k+1) \theta_{x}+\lambda \sin \Omega t\right]+ \\
& \frac{1}{2} I_{y}^{2}-\mu \cos \left[k \theta_{x}+\theta_{y}\right],
\end{aligned}
$$

where the $(\theta, I)$ variables are action-angle variables in each plane. Equivalences can easily be drawn between this model and that of the resonant beam-beam model used here to investigate modulational diffusion, which also gives the phase diagram in Figure 1. For example, $\epsilon \rightleftharpoons\left(2 \pi Q_{I}\right)^{2}$ (scaling of island tune), $\lambda \rightleftharpoons$ 


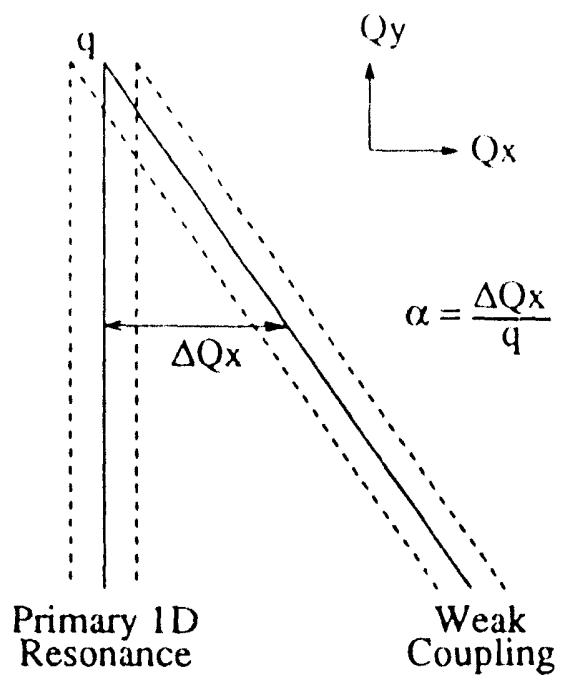

Figure 3: Resonance structure for modulational diffusion. $\alpha$ is the horizontal tune distance between the primary driving resonance and the secondary weak coupling resonance, scaled by the tune modulation depth $q$.

$\left(q / Q_{M}\right)$ (modulation strength), and $\Omega \rightleftharpoons\left(2 \pi Q_{M}\right)$ (modulation frequency).

One prediction of modulational diffusion theory is that this diffusive growth in the vertical dimension will scale as the square root of turn number. A diffusion coefficient can also be defined,

$$
D \equiv \frac{\left\langle\left[\Delta I_{y}(t)\right]^{2}\right\rangle}{2 T}
$$

where $\Delta I_{y}$ is the vertical action excursion from the initial action and $T$ is the time width of the averaging in turns. The averaging should be performed over a time $T$ short compared to the vertical diffusion time [so $\Delta I_{y} / I_{y}(t=0)$ is small] but long compared to the timescales of horizontal motion across the thick chaotic band. As the vertical tune is varied along the horizontal one-dimensional resonance, the proximity of the weak coupling resonance changes, as given by the dimensionless quantity

$$
\alpha \equiv \frac{\left.\mid Q_{x} \text { (weak coupling resonance }\right)-Q_{x} \text { (primary resonance) } \mid}{q} .
$$

A plot of the logarithm of the diffusion coefficient $D$ versus this scaled proximity $\alpha$ shows a series of descending plateaus and sudden drops ${ }^{1}$. This strange structure has sharp drops in $D$ at even integer values of $\alpha$ for the case where both the driving resonance and the coupling resonance are modulated, and it 


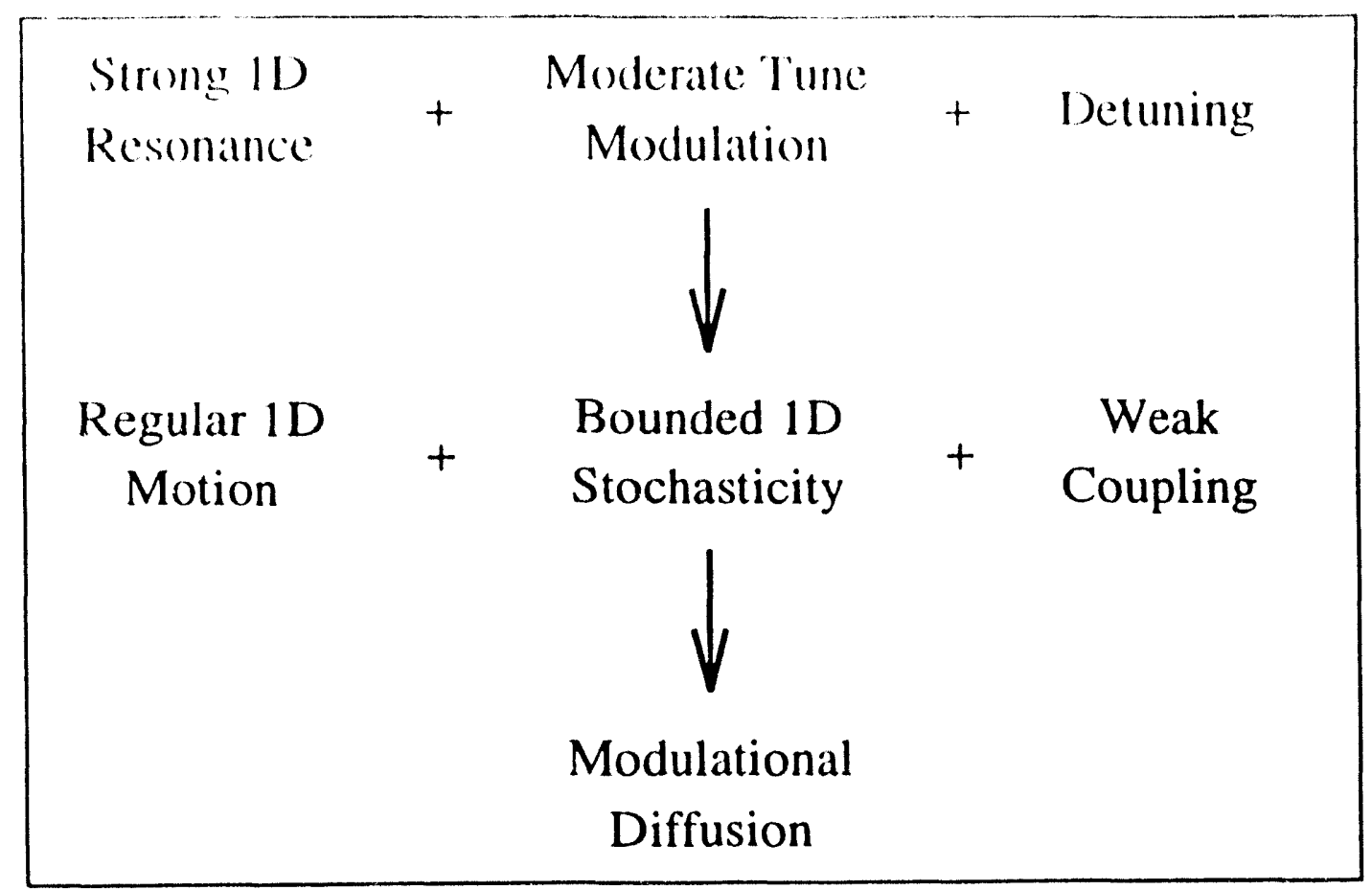

Figure 4: A diagrammed summary of the requirements for modulational diffusion.

is this sort of structure we attempt to qualitatively reproduce here within the operational framework of the Fermilab Tevatron.

\section{THE TEVATRON SITUATION AND AN OPERATIONAL MODEL}

In the Fermilab Tevatron during the 1992 collider run with separators, there were two strong beam-bean: interactions every turn - one at the CDF experimental site at ring location $\mathrm{B} 0$. and one at the D0 experimental site. The operating estimate of the linear beam-beam tune shift $\xi$ is approximately $\xi \approx 0.005$ per interaction, and with planned upgrades including the Fermilab Main Injector, this value may very well rise even further. ${ }^{7}$ With the exceptions of these beam-beam kicks and chromaticity-correction sextupoles (which are neglected for the sake of simplicity of the tracking model), the Tevatron is quite a linear machine, and so its transverse dynamics in this situation can be modeled simply using only linear phase advances and beam-beam kicks. Typical operating parameters for the 1992 collider run are listed in Table 1.

The base tunes of the Tevatron in typical collider run circumstances are $Q_{x 0} \approx 20.586$ and $Q_{y 0} \approx 20.575$, running at a horizontal tune between the 12 th and 5 th-order resonances. For the purposes of this study, however, a worst-case scenario is investigated, where the driving resonance for the horizontal stochasticity necessary for modulational diffusion is the 5th-order resonance 


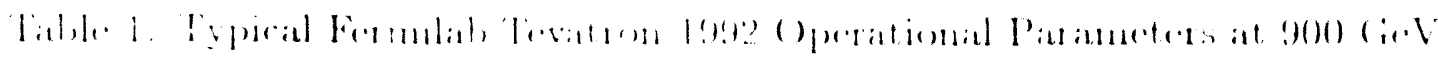

\begin{tabular}{|c|c|c|}
\hline 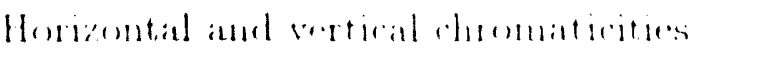 & $\left(\xi_{1}, \xi_{1}\right)$ & \\
\hline Typiral momentum off $\cdot 1 \cdot 1$ & $\Delta p / l$ & $0(0) 3$ \\
\hline Symelarotron (uncolulation) frepueney & $Q_{M}$ & $0.00)($ \\
\hline H. heia & $\xi$ & 0.0 \\
\hline Revolution freequency & frev & $47.7 \mathrm{kH}$ \\
\hline
\end{tabular}

and single particles are lanuched at a variety of vertical tunes along this resonance. If $\xi$ ever exceeds .009 with two collisions in the Tevatron, the available: space between the 12 th and the 5 th becomes too small for the entire beam, and a significant portion of the beam could be strongly affected by one of these resonances. The relevant portion of the tune plane diagram and the strange shape of the beam-bean footprint are shown in Figure 5.

The beam-beam force used here (within the tracking program Evol) uses the weak-strong approximation and assumes both beams have round Gaussian distributions of equal transverse size $\sigma$. For the horizontai beam-beam kick,

$$
\frac{\Delta x}{\sigma}=\frac{-4 \pi \xi}{3_{r}^{*} R^{2}}\left[1-e^{-R^{2} / 2}\right] \frac{x}{\sigma}
$$

where $x$ is the transverse pe.ition relative to the opposing beam center, $x^{\prime} \equiv$ $d x / d s, \beta_{x}^{*}$ is the beta function at the interaction point, and $R$ is the distance from the center of the opposing beam scaled to the beam size $\sigma$ :

$$
R \equiv\left(\frac{x}{\sigma}\right)^{2}+\left(\frac{y}{\sigma}\right)^{2}
$$

a kick similar to $(5)$ is seen in the vertical plane. Salient features relevant to this study can be noted:

- The variation of tune with amplitude (detuning) given by the bean-beam force is nonlinear and strongly coupled.

- From the form of $R$, even-order resonances are driven to first order in $\xi$. Odd-order resonances of order $N$ are driven as even-order resonances of order 2.2 .

- Resonance strengths vary with particle amplitude, or action.

- There is no beam-beam tuneshift or resonance driving at infinite amplitudes, so global motion of the unperturbed beam-bean system is stable.

The detuning is drastically different from the model of Equation 2, where there is explicitly no coupling other than the weak resonance, and the explicit variation of resonance strength with particle amplitude is also a difference between 


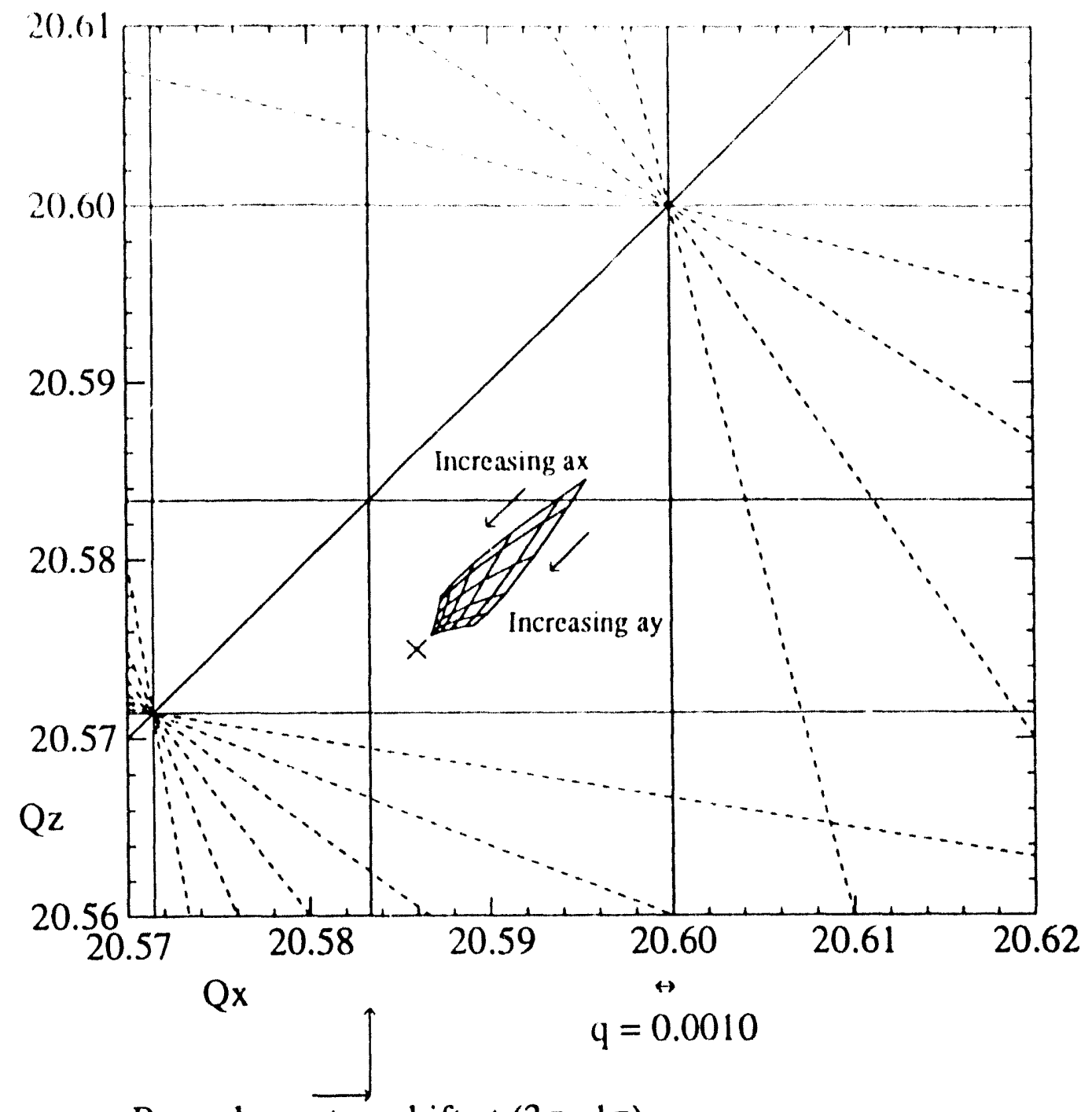

Beam-beam tuneshift at $(3 \sigma, .1 \sigma)$

Figure 5: The tune plane for typical Fermilab Tevatron 1992 collider operations, showing the $5 \mathrm{th}, 7 \mathrm{th}$ and 12 th-order resonances. The nominal operating tunes are indicated by the cross, and the bean-beam footprint is shown for $\xi=0.005$ with two collisions. Footprint contours of constant aniplitude range from $0.1 \sigma$ to $5.1 \sigma$ in $1 \sigma$ increments.

these two models. As vertical amplitude grows in the beam-beam situation, one of two mechanisms wiil halt modulational diffusion: the vertical anplitude grc wth will either pull the horizontal tune off the primary driving resonance ur it will suppress the coupling resonance strength. It remains to be conclusively 


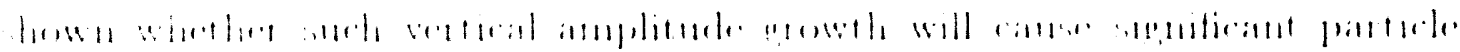

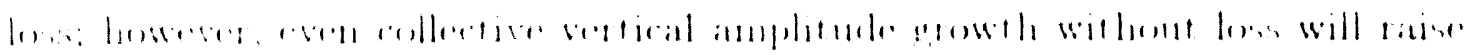

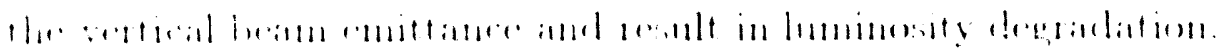

\section{SIMILATION RESULTS}

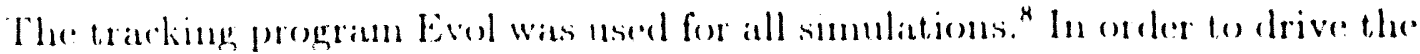
Stherder resonance strongly for the worst cibse sechatro, a small horzontal 0.10 beam-beam offset was included; closecherbit alignment errors of this magnitude at the collision points are quite possible. Tracking this lattice with no tune modulation with the beam-beam tuneshift given in Table 1 on the $Q_{x}=20.6$ resonance tinds an island tune of $Q_{l}=1.51 \times 10^{-3}$. Since the synchrotron frequency of the Tevatron at this energy is approximately $Q_{M}=7.8 \times 10^{-4}$ (with a neriod $T_{M}=1 / Q_{M}=1280$ turns), the chastic region of the tune modulation parameter space in Figure 1 is quite accessible for moderate tune modulation depths $q$. In this study a tune modulation depth $q=0.0010$ was used, present only in the horizontal plane for comparison to the results originating in the similarly modulated Hamiltonian of Equation 1. This tune modulation amplitude corresponds to a horizontal chromaticity of about 3 units with a momentum offset $\Delta p / p$ of $3 \times 10^{-4}$, realistic values for the Tevatron.

To establish the timescales of the relevant amplitude growth nechanisms, the maximum vertical amplitude was recorded for single particles launched at the above initial conditions over a mesh on the tume plane, for tracking times ranging from 10 to $10^{4}$ synchrotron periods. The tune mesh limits used were the same as those shown in the tume plane diarram, Figure 5, and the results of this tracking are shown in Figures 6a-6d. A quite definits vertical amplitude growth is seen near the intersections of the $Q_{x}-Q_{y}$ and $5 Q_{x}$ resonances that evolves over timescales of thousands of synchrotron periods, consistent with the timescales of modulational diffusion. Such growth is completely absent with modulation turned off $(q=0)$, where only amplitude growth on the $Q_{x}-Q_{y}$ resonance is seen due to energy exchange between the unbalanced horizontal and vertical amplitudes; this is a conclusive indication that the modulation drives this vertical amplitude growth. The growth also seems to have a structure along the horizontal resonance, consistent with the moduational diffusion model expectations of the dependence of the amplitude growth mate on distance from the nearest coupling resonance. Some growth also appears on the $3 Q_{x}+2 Q_{y}$ resonance; however, the structure along this resonance is quite minimal in comparison to the vertical amplitude growth near the previously mentioned intersection of $Q_{x}-Q_{y}$ and $\zeta Q_{x}$ resonances.

Once the timescales of amplitude growth have been established, there remains the question of how the vertical amplitude evolves with time. It has already been mentioned that classical diffusion predicts that the vertical amplitude will grow 

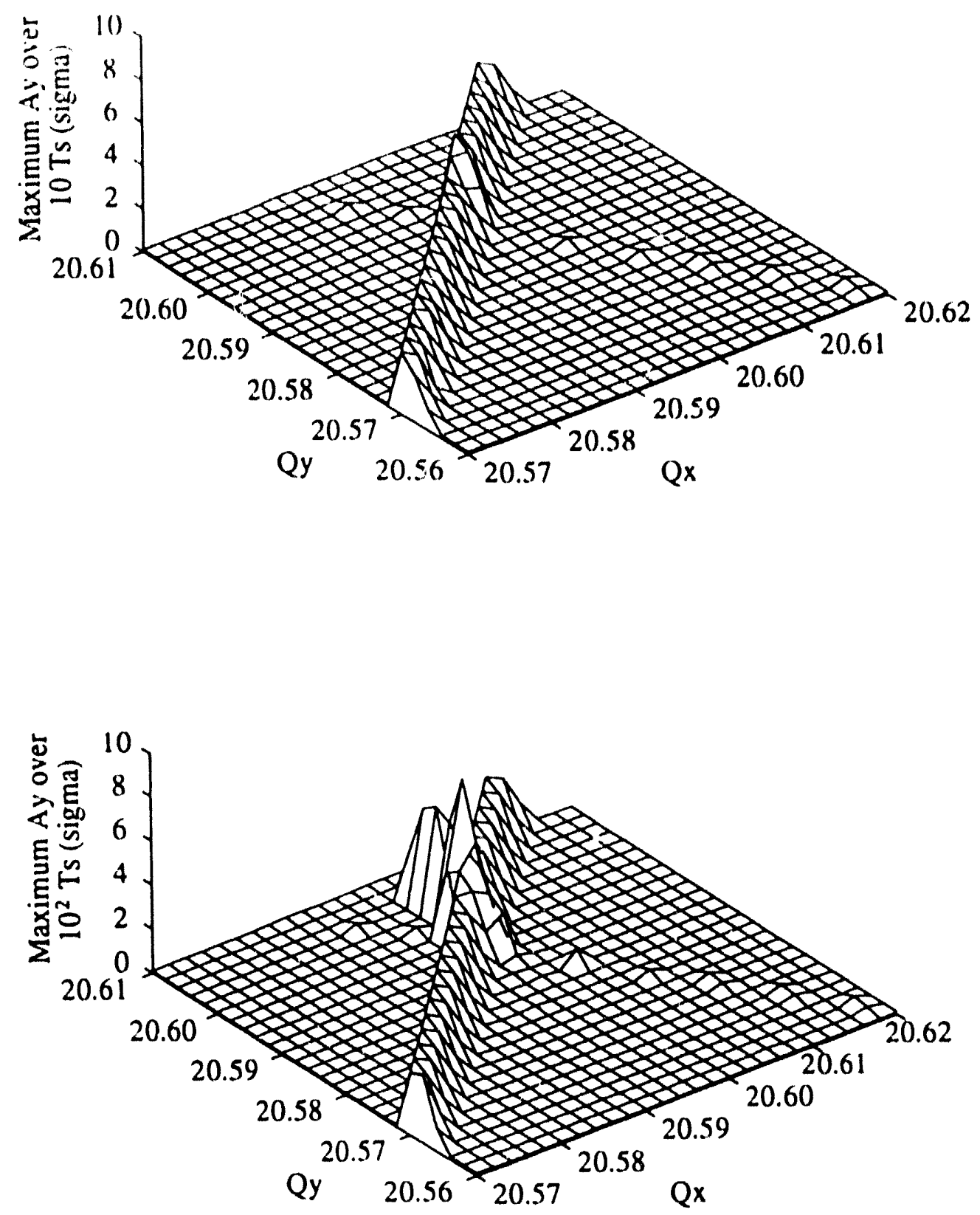

Figures 6a and 6b: Maximum vertical amplitudes of single particles with initial vertical amplitudes $0.1 \sigma$, tracked over 10 and 100 synchrotron periods. Horizontal particle amplitude is $3 \sigma$, inside a chawtic band. 

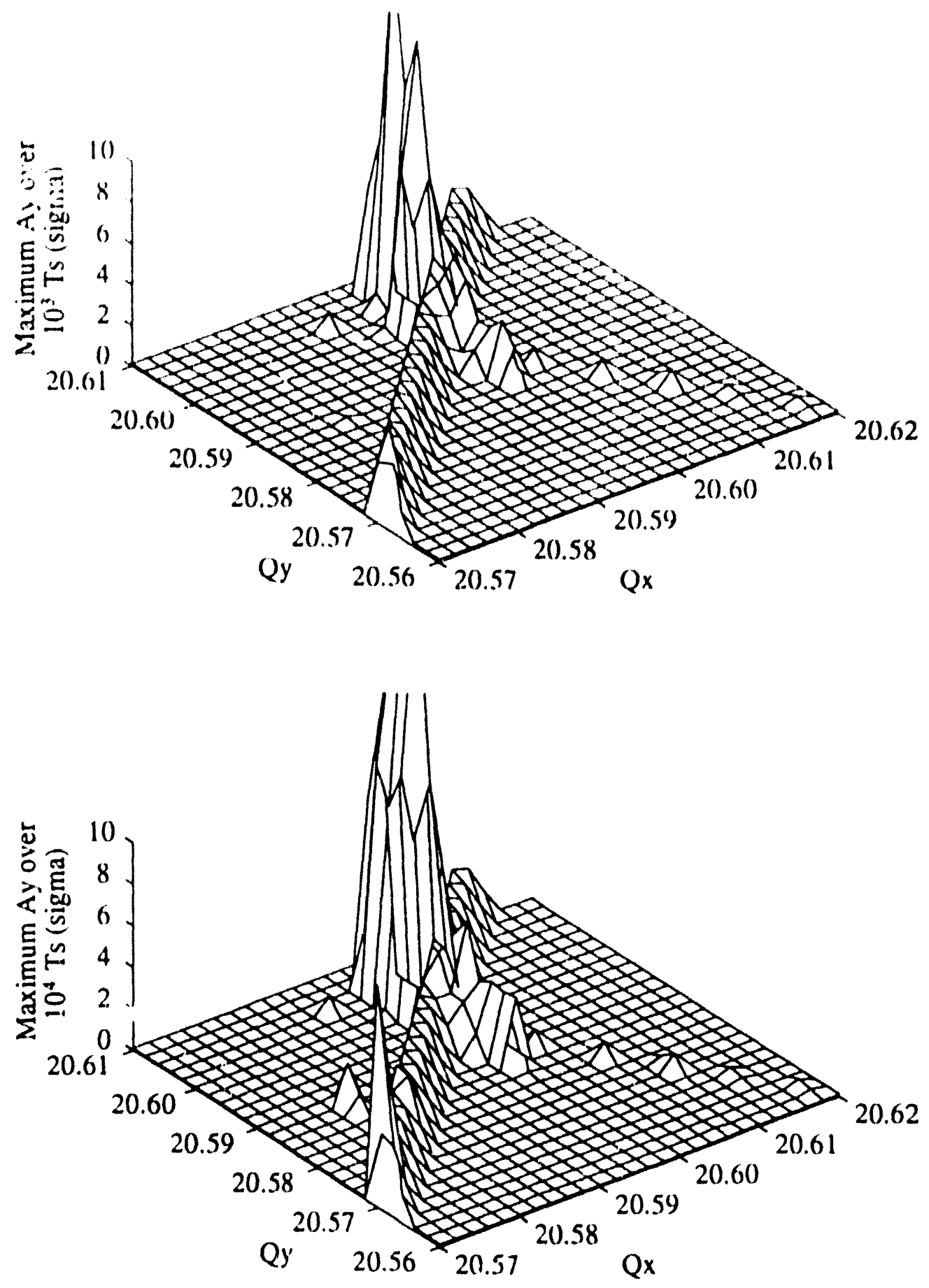

Figures 6c and 6d: Maximum vertical amplitudes of single particles with initial vertical amplitudes $0.1 \sigma$, tracked over $10^{3}$ and $10^{4}$ synchrotron periods. 


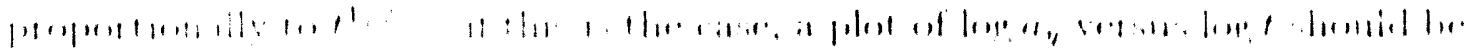

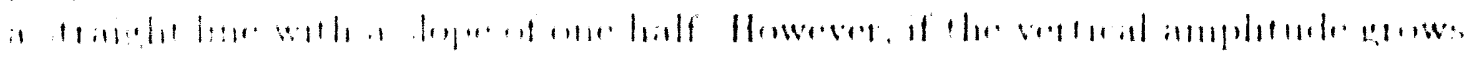

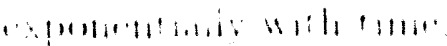

$$
\|,(t) \quad\| 11, " t
$$

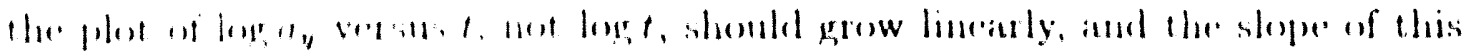

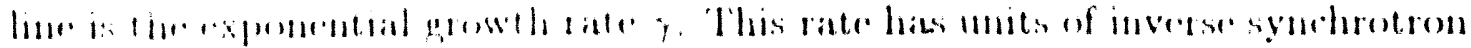

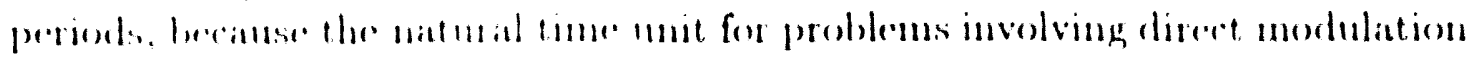
is the mondulation pretionl.

To investigate the vertiral anuplitude cvolution, particles were lanuched with Lorizontal ampliturle of $3 \mathrm{\sigma}$, with hisse tume: $Q_{x 0}=20.597$ (directly on the $5 Q_{x}$ resomanere whth the heam-heam tume shift) and vertical amplitude of $0.1 \%$ with varions base tuncs. Tratking was stopped when either $10^{4}$ synchroten feriods had beren tracked (correspomding to nearly 5 minutes of real pauticle coolution), or the vertical ampliturle had reateled $1 \sigma$. The one-sigma vertical cutoff was introduced heranse the influence of the vertical motion on the horizontal motion within the stochastic hand was expected to become non-negligible at moderate vertical anplitudes; both the beam-beam detuning and resonance strengths decroase as vertical anditules increasies.

Figure i shows three eximples of vertical amplitude evolution over relietively long timescales, each plotted on log-linear and log-log scales. It is clear from rxanning these evolutions (as well as those of many other particles at different distances a from the nearby weak compling resonance $4 Q_{x}+Q_{y}$ ) that the vertical amplitude is growing as an exponential of time, not a power law as one wouid expect from standard diffusion phenomenology. It has been suggested ${ }^{9}$ that this behavior maty be explained hy the dependence of resonance strengths on particle: amplitude ... the change in amplitude creates a changing resonance strength which feeds back upon the amplitude growth, creating exponential growth.

The exponential growth eoefticient $\gamma$ can now be plotted versus the scaled distance to the weak coupling resonance as one varies the vertical tune along tine $5 Q_{x}$ resonance to investigate whether there is any structure present. Since $\gamma$ is expected to vary over many orders of magnitude, we instead plot $\log \gamma$ versus a; a can be directly determined from the vertical base tune $Q_{\text {yo }}$ via

$$
a=\frac{20.60-Q_{y 0}}{5 q}
$$

when considering the $4 Q_{x}+Q_{y}$ resonance to be the source of weak coupling. Other resonances such as $Q_{x}-Q_{y}$ and $3 Q_{x}+2 Q_{y}$ are also nearby, but are farther away in horizontal tune distance $\alpha$ than this resonance, as cas be seen in Figure 5. The exponential growth rate $\gamma$ is measured from a standard linear fit of tracked $\log a_{y}$ versus time data. Figure 8 shows this data; note the two distinct "plateaus" and the sudden drops in the growth rate at $\alpha=2$ and $\alpha=3$. 

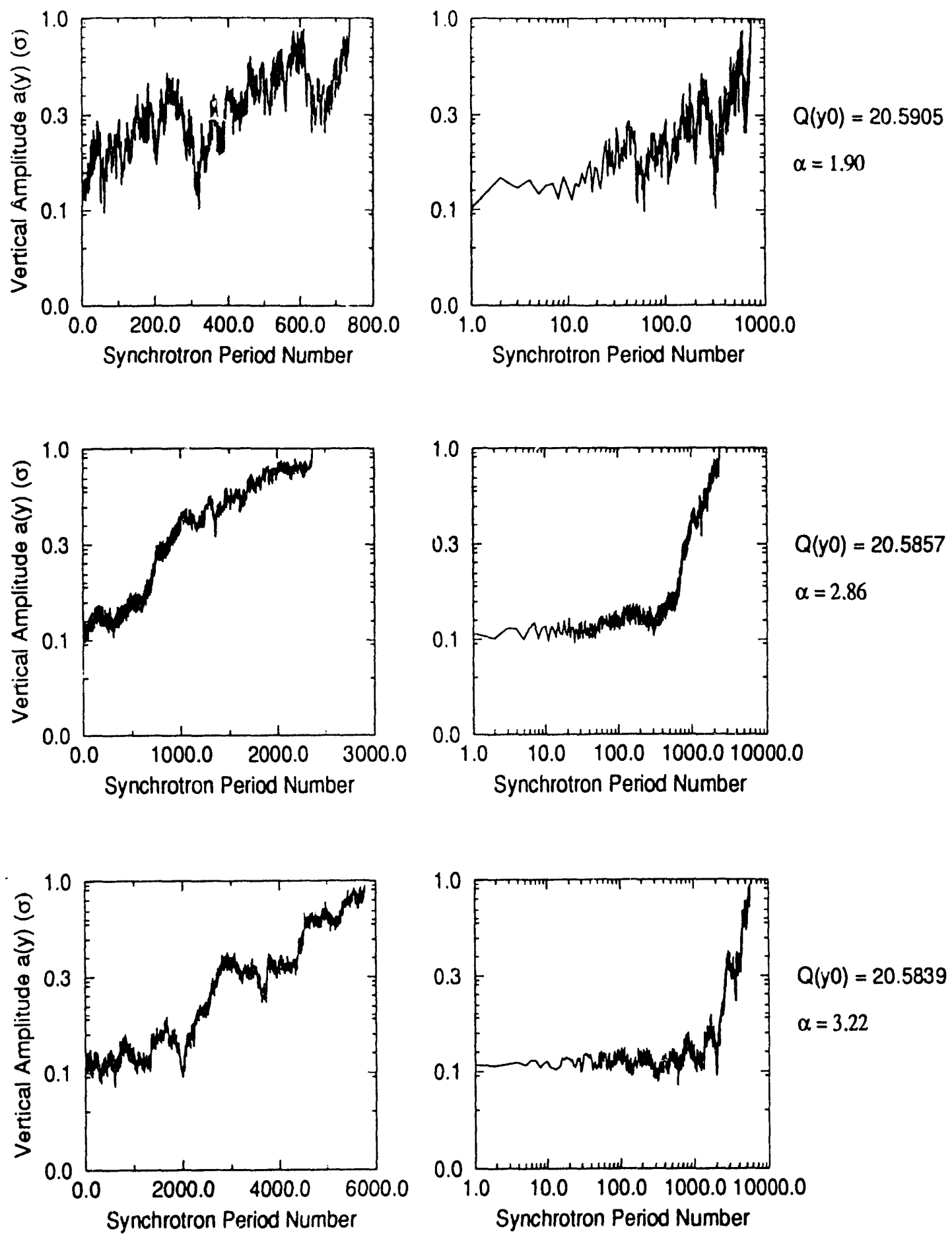

Figure 7: Character of vertical amplitude growth for particles launched within a horizontal stochastic band, and at initial vertical amplitude $0.1 \sigma$. Tracking was stopped when the vertical amplitude reached $1 \sigma$; note the differing growth timescales. 


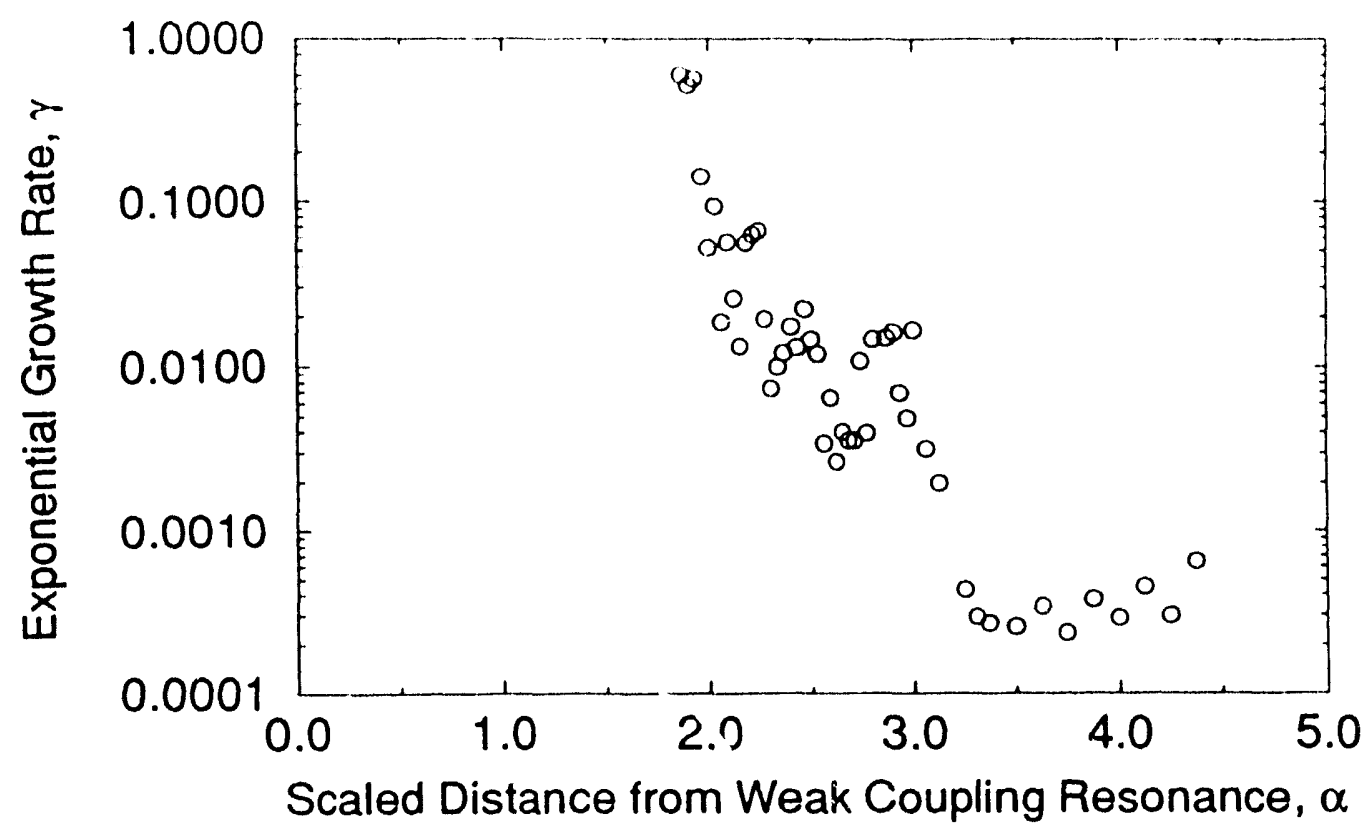

Figure 8: Exponential vertical amplitude growth rate $\gamma$ plotted versus the scaled distance $\alpha$ from the $4 Q_{x}+Q_{y}$ resonance.

\section{CONCLUSIONS AND FUTURE DIRECTIONS}

Modulational diffusion has been investigated within a simple model of the beam-beam interaction in the Fermilab Tevatron collider. Realistic operational parameters indicate that particles subject to horizontal stochasticity, or naively those that are within the tune modulation depth distance in horizontal tune of the $5 Q_{x}$ resonance, experience modulational diffusion that causes their vertical amplitudes to grow exponentially over timescales of thousands of synchrotron periods, or millions of turns, leading to possible long-term particle loss. The rate of this amplitude growth is also dependent on proximity of nearby coupling resonances, and shows a structural dependence similar to those of previous modulational diffusion studies. However, the vertical amplitude growth is not root-time as naively predicted in these models where resonance strengths are not action-dependent.

Under current operating conditions in the Fermilab Tevatron collider, no particles are expected to be affected by the $5 Q_{x}$ resonance this severely unless the horizontal tune drifts upwards, dragging particles into the fifth, or the linear beam-beam tune shift $\xi$ increases. However, with future luminosity upgrades, this tune shift per crossing will almost certainly rise, and the operational space used in past runs may not be large enough to accomodate the entire tune spread of the beam, with a significant portion of the beam becoming influenced by the horizontal 7 th and 5 th and vertical beam blowup as a result. This could lead 
to luminosity degradation and intensity loss over a collider store as the beam size grows. Also, these effects, were they present in an actual collider, could be difficult to diagnose dine to their slow growth nature.

Future studies should be twofold. First, a concrete theoretical structure of modulational diffusion should be investigated to conclusively show that in the case of amplitude-dependent coupling resonance strengths, vertical amplitude growth is exponential instead of root-time as in classical models. Secondly, the statistical nature of this amplitude growth should be investigated to see what observable effects such a mechanism could have on the beam size (and thus luminosity) evolution over time.

Another tracking model might be used to investigate the amplitude growth mechanism once a theoretical framework is in place, to avoid the rather complex detuning coupling of the beam-beam force. A lattice with sets of octupoles (which control detuning independently to first order in their strength in each transverse plane) and a decapole (which drives the horizontal $5 Q_{x}$ resonance to first order) has already been developed for other tune modulation investigations. ${ }^{5}$ Tracking with this lattice has several distinct advantages - motion at large particle amplitudes is no longer stable, so no ad hoc aperture needs to be introduced, and parameters for a Hamiltonian description as in Equation 1 can easily be found to first order in the individual magnet strengths.

\section{REFERENCES}

1. B.V. Chirikov, M.A. Lieberman, F. Vivaldi and D.L. Shepelyanski, "A Theory of Modulational Diffusion", Physica 14D, 298 (1985).

2. F. Vivaldi, "Weak Instabilities in Many Dimensional Hamiltonian Systems", Rev. Mod. Phys. 56, 737 (1984).

3. A.J. Lichtenberg and M.A. Lieberman. Regular and Stochastic Motion (Springer, New York, 1983).

4. S. Peggs, "Hamiltonian Theory of the E778 Nonlinear Dynamics Experiment", SSC-175 (April 1988).

5. T. Satogata, Ph.D. Thesis. Northwestern University (1993).

6. T. Satogata et. al. , "Driven Response of a Trapped Particle Beam", Phys. Rev. Lett. 68, 1838 (1992).

7. Steven D. Holmes, "Achieving High Luminosity in the Fermilab Tevatron", Proceedings of the 1991 IEEE PAC, 2986 (1991).

8. S. Peggs, "Hadron Collider Behavior in the Nonlinear Numerical Model Evol", Particle Accelerators 17, 11 (1985).

9. O. Brüning, private communication at this workshop. 

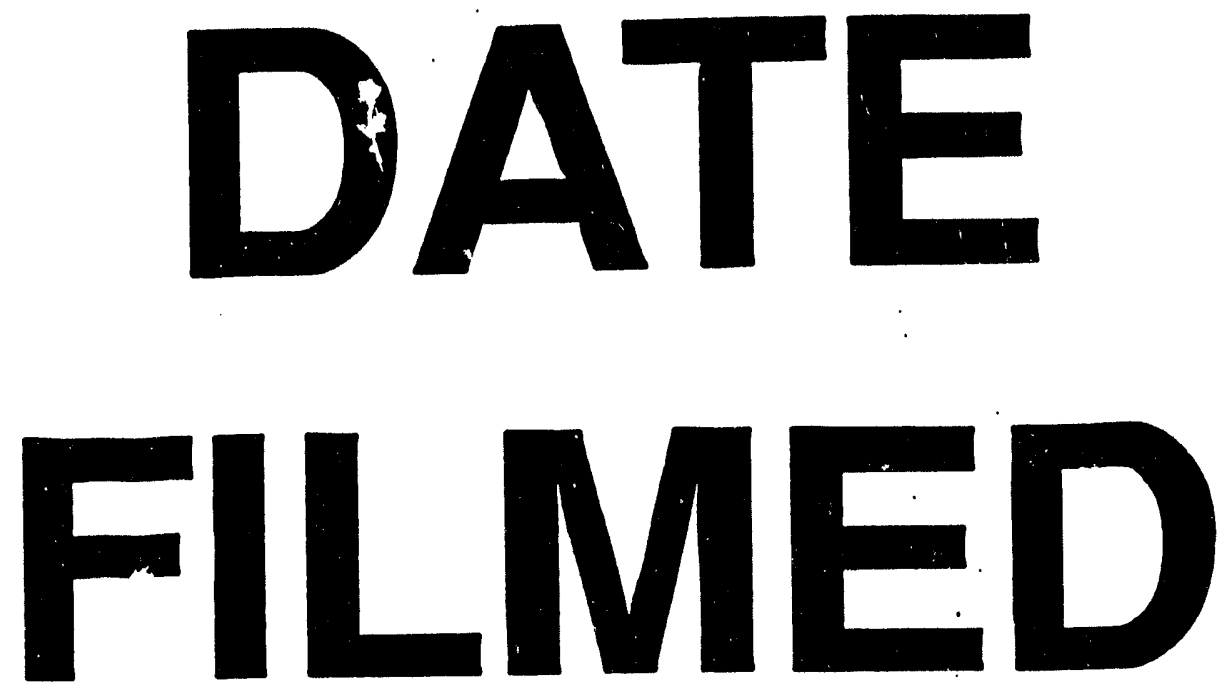

$11 / 5 / 93$
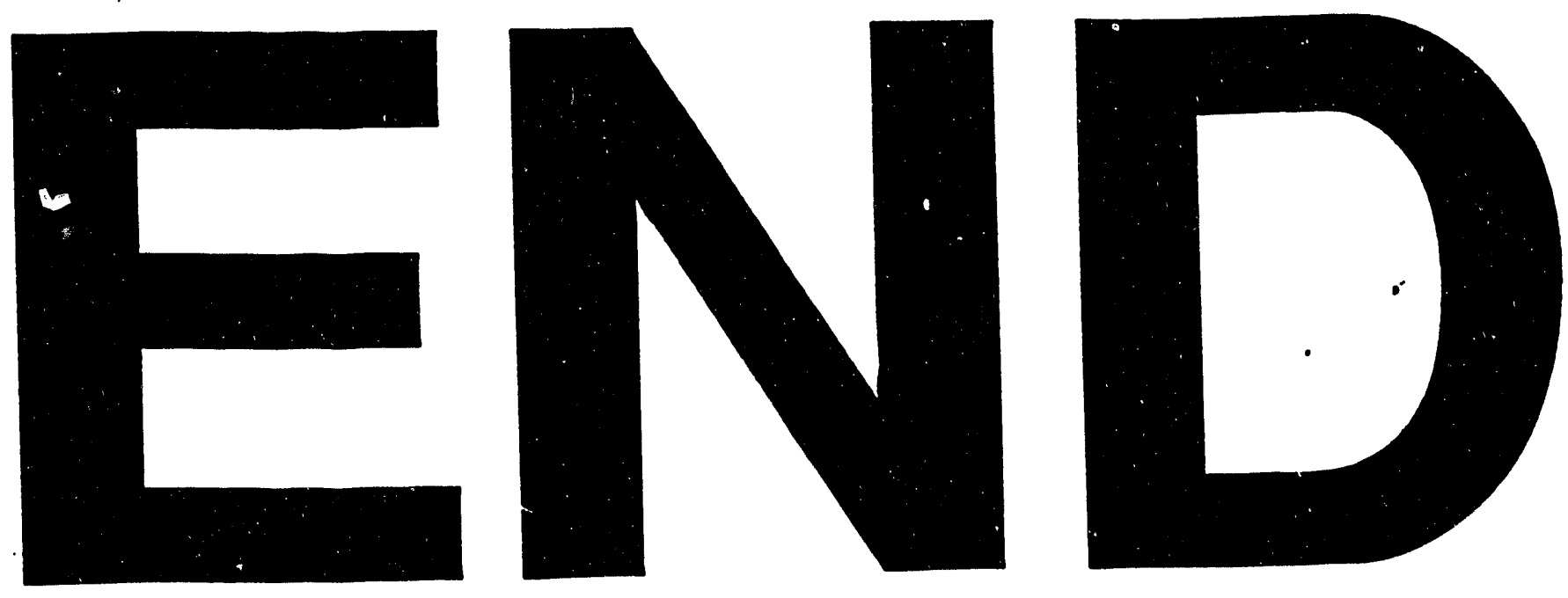


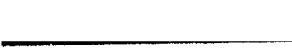

